\title{
Du conditionnel comme constructions ou la polysémie du conditionnel ${ }^{1}$
}

\author{
Adeline Patard \\ Université Caen Normandie
}

\section{Résumé}

Les approches du conditionnel français (désormais COND) sont généralement monosémistes (le COND possède un sens unique), souvent compositionnelles (il est composé de deux morphèmes $/ \mathrm{R} /$ et $/ \varepsilon /$ ) et implicitement non-conventionnelles (les valeurs interprétées dans les énoncés ne sont pas conservées dans les connaissances linguistiques du locuteur). L'article propose une conception alternative du COND polysémique, non-compositionnelle et conventionnelle -qui se fonde sur la notion de construction des grammaires de construction (Goldberg 1995, 2006). Selon cette conception, le COND correspond à trois constructions en français, de sens temporel, modal et évidentiel. Cette approche est appuyée par une analyse diachronique qui montre notamment sa double origine étymologique en latin.

\begin{abstract}
Approaches to the French conditionnel (henceforth COND) are generally monosemist (COND has one single meaning), often compositional (it is composed of two morphemes $/ \mathrm{R} /$ and $/ \varepsilon /$ ) and implicitly non-conventional (the values interpreted in utterances are not stored in the linguistic knowledge of the speaker). The article suggests an alternative conception of COND - polysemic, non-compositional and conventional - which is based on the notion of construction developed by construction grammars (Goldberg 1995, 1996). According to this conception, COND corresponds to three constructions in French, with a temporal, modal or evidential meaning. This approach is further supported by diachronic data that notably show the double etymological origin of COND in Latin.
\end{abstract}

\section{Introduction}

Le conditionnel (désormais COND) est une forme verbale présentant de nombreux contextes d'emploi avec des interprétations aussi bien temporelles que modales (d'où la question posée par la grammaire traditionnelle : temps ou mode ?). On lui reconnaît en général trois ou quatre classes de valeurs actualisées en discours. Citons pour illustration les quatre «emplois canoniques» distingués par Dendale $(2001)^{2}$ :

Ultérieur du passé (ou « futur du passé »)

1. Enfin, le M..P.. [...] a dit qu'il repasserait me chercher le lendemain et il m'a oublié, bien sûr. (Azzopardi 2011)

Emprunt (ou «évidentialité » ou « médiativité »)

2. Le recours aux biocarburants serait un remède pire que le mal. (Bres et al. 2012)

\footnotetext{
${ }^{1}$ Je remercie J. Bres ainsi que les deux relecteurs anonymes de Langue française pour leurs remarques pertinentes et constructives qui ont permis d'améliorer l'article.

${ }^{2}$ Nous avons adapté les dénominations de chaque emploi selon l'usage que nous en faisons dans l'article.
} 
Eventualité (ou « hypothèse »)

3. S'il ne parvenait pas [...] à convaincre qu'il est déterminé à aller jusqu'au bout, l'opinion publique le prendrait très mal. (Haillet 2002)

Atténuation (ou « bémolisation » ou « mitigation »)

4. Je voudrais rencontrer le président. (Vetters 2001)

Le COND a récemment suscité l'intérêt des linguistes avec des hypothèses convergeant sur l'origine de cette « polysémie » et prolongeant une certaine tradition grammaticale (voir notamment Guillaume 1929 et Damourette et Pichon 1911-1936/ 1970). L'approche proposée est généralement :

- une approche monosémiste: le COND se caractérise en langue par un invariant sémantique temporel, énonciatif et/ou modal (parmi d'autres Abouda 2001, Korzen et Nolke 2001, Vet et Kampers-Manhe 2001, Moeschler et Reboul 2001, Wilmet 2001, Vetters 2001, Haillet 2002, Kronning 2002, Provôt 2011, Azzopardi 2011, Bres et al. 2012 , etc.) ;

- $\quad$ une approche compositionnelle : cette valeur est obtenue par la somme des valeurs des morphèmes $/ \mathrm{R} /$ et $/ \varepsilon /$ qui composent le COND (voir par ex. Touratier 1996, Gosselin 1999, Vetters 2001, Camussi-Ni 2006, Azzopardi 2011, Bres et al. 2012, Abouda 2016) ;

- une approche a priori non-conventionnelle fondée sur une conception sémantique « holiste» ou «dynamique »: les sens co(n)textuels sont le résultat d'une interaction entre l'invariant sémantique du COND et son co(n)texte; autrement dit, les sens co(n)textuels sont reconstruits de façon $a d$ hoc à chaque énoncé, les valeurs interprétées ne sont donc pas a priori conventionnelles, i.e. conservées dans les connaissances linguistiques du locuteur (Abouda 2001, Gosselin 2001, Korzen et Nolke 2001, Vetters 2001, Bres et al. 2012, Abouda 2016).

Le but de cet article est de montrer qu'il est possible de défendre une approche alternative du COND - polysémiste, non-compositionnelle et conventionnelle - en se fondant sur la notion de « construction » développée dans les grammaires de construction. Nous tâcherons de montrer que cette approche est plus directement compatible avec les données diachroniques, et notamment avec l'origine étymologiquement distincte des deux emplois canoniques pressentis comme «plus fondamentaux ", à savoir les emplois à valeur d'ultériorité dans le passé et d'éventualité (Dendale $2001: 9)$.

\section{L'approche monosémiste}

\subsection{Un invariant sémantique}

Les analyses monosémistes récentes du COND postulent un invariant sémantique abstrait qui serait à l'origine de toutes les interprétations rencontrées dans les énoncés. Ce postulat «abstractionniste » (Goldberg 1995 : 34), qui repose sur le principe d'iconicité « une forme, un sens », est souvent adopté pour l'élégance et l'apparente simplicité de l'analyse qu'il permet. L'invariant sémantique du COND est alors recherché dans l'un de emplois canoniques précités ${ }^{3}$ qui instancierait plus directement son sens unique.

Pour l'approche temporelle, l'invariant sémantique du conditionnel est celui qui s'exprime dans son emploi d'ultérieur du passé (cf. (1)). Le COND est alors défini comme un véritable temps de l'indicatif (et non comme un mode sui generis) qui signifie un double rapport temporel: l'antériorité d'un point ou intervalle de référence ( $\mathrm{R}$ de Reichenbach) par rapport au présent du locuteur et l'ultériorité de la situation dénotée par rapport à ce point ou intervalle passé (voir p.

\footnotetext{
${ }^{3}$ A l'exception de l'emploi d'atténuation. Cet emploi n'est d'ailleurs pas reconnu par une partie des linguistes (voir p. exemple Haillet 2002, Kronning 2002, Caudal et Vetters 2005).
} 
ex. Gosselin 2001, Vet et Kampers-Manhe 2001, Wilmet 2001, Azzopardi 2011, Bres et al. 2012). Cette hypothèse sémantique est souvent appuyée par une analyse composititionnelle du COND : le rapport d'antériorité serait signalé par le morphème /E/ issu de la forme écrasée de habebam en latin, faisant ainsi du COND un temps du passé comme l'imparfait ; quant au rapport d'ultériorité, il serait indiqué par le morphème /R/ issu de la flexion -re/ri de l'infinitif latin, le COND serait donc également un temps «futur» ou «ultérieur» (Wilmet 2001, Azzopardi 2011, Bres et al. 2012). La définition temporelle du COND est aussi souvent mise en relation avec un contenu dialogique (ou polyphonique) qui en découlerait directement : le COND nécessite de se représenter un énonciateur secondaire passé qui puisse envisager ultérieurement la situation dénotée (Bres 2009, Azzopardi 2011, Bres et al. 2012).

Pour l'approche énonciative, l'invariant sémantique du COND se perçoit dans son emploi d'emprunt qui serait l'emploi «basique » ou «par défaut ( (Abouda 2001, Kronning 2002). Dans cette perspective, le COND est conçu comme un marqueur médiatif ou évidentiel, avant d'être temporel ou même modal (voir aussi Korzen et Nølke 2001, Haillet 2002 et 2003). C'est parce qu'il indique que l'information communiquée dans l'énoncé n'est pas prise en charge par le locuteur, mais empruntée à une source énonciative secondaire, que le COND peut contextuellement renvoyer à un locuteur passé ou exprimer des valeurs modales comme l'incertitude. Cette approche converge avec une conception inactuelle du COND s'inspirant de Damourette et Pichon (19111936/ 1970) qui repose sur une analyse componentielle de sa morphologie (Vetters 2001, Abouda 2016) : en tant que "futur de l'imparfait », le COND dénoterait l'ultériorité (sens du morphème /R/ du futur), et exprimerait une disjonction avec l'actualité présente du locuteur (sens du morphème $/ \varepsilon$ / de l'imparfait).

Pour l'approche modale ou de la « conditionnelle sous-jacente généralisée », l'emploi standard $\mathrm{du}$ COND est celui des conditionnelles hypothétiques en [si p, (alors) q] (Korzen et Nølke 2001 ${ }^{4}$, Moeschler et Reboul 2001). L'invariant sémantique serait alors proprement "conditionnel », il soumettrait le contenu de l'énoncé ou l'acte de langage à une condition toujours sous-entendue et pouvant être explicitée par une protase. Cette dernière approche, qui est semble-t-il minoritaire aujourd'hui, s'inscrit dans la tradition linguistique du COND comme mode.

\subsection{Calculs interprétatifs des « effets de sens »}

Les analyses monosémistes du COND s'appuient plus ou moins explicitement sur une conception « holiste » ou «dynamique » de la construction du sens (cf. Gosselin 1996a et 1996b, Victorri et Fuchs $1996^{5}$ ou Jalenques ici-même pour une présentation). Selon cette conception, les interprétations attachées au COND sont des « effets de sens » émergeant de l'interaction entre une valeur stable et abstraite et les apports sémantiques du co(n)texte, position illustrée par la citation suivante :

Notre hypothèse est donc qu'on peut discerner une signification stable du conditionnel (...) et que la diversité des valeurs observées s'explique par des propriétés du contexte (effets de sens). (Korzen et Nølke 2001 : 126)

Le linguiste se donne ainsi pour tâche de rechercher, à partir de la diversité des « effets de sens », le sens unique du COND et de rendre compte de ses interprétations par les particularités contingentes du co(n)texte. Chaque interprétation co(n)textuelle est alors en principe calculable à partir du

\footnotetext{
${ }^{4}$ L'analyse de Korzen et Nølke (2001) combine des éléments des deux dernières approches : le COND exprime un déplacement par rapport aux coordonnées du centre énonciatif (approche énonciative-inactuelle) qui peut être formulé métalinguistiquement par une subordonnée conditionnelle : si on se place à $R$, c'est le cas que $P$ (approche modale).

${ }^{5}$ Notons que Gosselin (1996a et 1996b) et Victorri et Fuchs (1996), se fondant sur le constat d'une polysémie (contextuelle) généralisée, présentent leur approche comme polysémiste. Toutefois, comme ils reconnaissent une valeur en langue unique pour chaque marqueur, on peut les compter ici parmi les auteurs défendant une approche monosémiste.
} 
signifié du COND et des éléments de sens apportés par le co(n)texte. Selon l'approche adoptée (temporelle, énonciative et/ou modale) et selon l'emploi visé, deux types de calcul interprétatifs peuvent intervenir.

- L'élaboration du sens : le co(n)texte vient étoffer ou saturer le schéma sémantique abstrait du COND. Cette analyse suppose que les interprétations co(n)textuelles entretiennent une relation d'hyponyme-hyperonyme avec l'invariant sémantique. Les approches énonciative et modale semblent avoir principalement recours à ce type de calcul pour expliquer les différentes interprétations du COND (voir entre autres Abouda 2001, Korzen et Nølke 2001, Haillet 2002 et 2003, Kronning 2002) ${ }^{6}$.

- La dérivation du sens (au sens large) : ce type de procédure est invoqué lorsque l'interprétation finale n'apparaît pas comme un hyponyme de l'invariant sémantique. Selon cette procédure, l'interprétation standard qui instancie directement l'invariant sémantique est "modulée» sous l'action du contexte (les auteurs parlent souvent d' «interaction» avec le contexte). En conséquence, l'interprétation du COND est ajustée aux conditions contextuelles pour produire le sens visé par l'énoncé. Ce type de procédure peut prendre différentes formes : allègement du sens ou neutralisation de traits sémantiques, glissement de sens, action d'opérateurs, inférence, etc. Les procédures de dérivation sont plus ou moins explicitement exploitées dans le cadre de l'approche temporelle pour rendre compte des interprétations non-temporelles du COND (Gosselin 1999 et 2001, Vet et Kampers-Mahne 2001, Wilmet 2003, Azzopardi 2011, Bres et al. 2012).

Ces procédures, qui permettent d'articuler invariant sémantique et "effets de sens », peuvent toutefois être sujettes à discussion. D'un côté, ne recourir qu'à la procédure d'élaboration, comme le font largement les approches énonciative et modale, suppose d'isoler un invariant abstrait très sous-spécifié. Or la démarche peut se révéler problématique, car un signifié très général encourt toujours le risque d'être trop puissant et non-opératoire :

Elle conduit, en effet, au terme de la procédure d'abstraction (ou induction des traits nécessaires) à les «vider » presque intégralement de leur signification, ou à ne pouvoir décrire cette signification de base que de façon purement négative (par ce qu'elle ne peut exprimer), de sorte qu'il devient extrêmement difficile de définir différentiellement les valeurs des marqueurs aspectuo-temporels, bien que ceux-ci ne soient pas librement interchangeables en contexte. De plus, la pauvreté de la signification en langue qui leur est reconnue rend pratiquement impossible le calcul de leurs effets de sens en contexte. (Gosselin 1996a : 114) ${ }^{7}$

D'un autre côté, les procédures de dérivation sont critiquables si «elles se font au coup par coup au moyen d'analyses $a d$ hoc qui nécessitent un certain talent de la part du linguiste, car elles s'avèrent parfois très acrobatiques » (Confais $1990: 53)^{8}$. On peut en particulier contester le bienfondé des procédures qui prévoient un allègement du sens ou la neutralisation de traits sémantiques :

L'explication par neutralisation consiste à dire que ce temps a perdu l'un de ses traits définitoires (...) Or, cela n'est pas sans poser des problèmes : si le sème disparait, pourquoi en faire un trait définitoire et surtout un sème premier? (Abouda $2004: 151)^{9}$

${ }^{6}$ Les analyses en termes d'élaboration du sens sont aussi développées dans l'approche temporelle pour rendre compte de certains emplois (cf. Gosselin 2001, Azzopardi 2011, Bres et al. 2012).

${ }^{7}$ Pour d'autres critiques d'un signifié trop vague ou général dans le domaine aspectuotemporel, voir aussi Berthonneau et Kleiber 1994 (p. 64), Gosselin 1999 (p. 31), Vetters 2001 (pp. 195-196), Patard 2007 (p. 234).

${ }^{8}$ Voir Barbazan 2006 (section 4 .1) pour une critique plus détaillée.

${ }^{9}$ Voir aussi les critiques de Gosselin (1996a : 114-115) et de Bres (2003: 102). 
L'édification d'une théorie sémantique satisfaisante dans un cadre monosémiste - i.e. permettant de dégager un invariant sémantique du COND et de l'articuler à ses effets de sens - est donc une tâche particulièrement délicate et les démarches mises en œuvre ne sont pas exemptes de travers. Des solutions alternatives ont été proposées dans le domaine du temps verbal, qui tentent de préserver l'hypothèse monosémiste (cf. p. ex. la démarche hypothético-déductive de L. Gosselin (1996a et 1996b)).

On peut toutefois s'interroger sur le fait que l'hypothèse monosémiste est habituellement privilégiée dans le cas des temps verbaux (et des formes grammaticales en général). Pourquoi celleci serait préférable à l'hypothèse polysémiste ? Ce choix semble guidé par une conception du langage marquée par une économie de la langue: un minimum d'informations encodé dans la mémoire à long-terme mais un calcul interprétatif qui demande inversement plus d'effort. $A$ contrario, l'hypothèse polysémiste suppose une économie dans le calcul interprétatif : davantage de significations sont directement encodées en langue, ce qui signifie moins de procédures d'interprétation et donc une moindre mobilisation de la mémoire de travail. Autrement dit, l'hypothèse polysémiste implique un traitement moins couteux et plus «automatique» ou «procédural ». Ainsi donc, aucune des deux hypothèses ne paraît préférable à l'autre in abstracto (économie cognitive d'un côté et économie de traitement de l'autre). Seuls des travaux en psycholinguistique permettraient d'avancer sur cette question précise.

Dans notre article, nous souhaitons explorer l'hypothèse polysémiste, souvent délaissée sans explication, et considérer le COND comme un ensemble de constructions (section 3). Mais, auparavant, nous voudrions envisager la question sémantique sous l'angle de la diachronie.

\subsection{Dimension diachronique}

Il nous semble éclairant, pour approfondir la discussion, d'examiner quelles sont les implications diachroniques des approches évoquées ci-dessus. En effet, une théorie ou une analyse, pour synchronique qu'elle soit, a souvent des incidences sur les scénarios possibles en diachronie, ce dont les linguistes n'ont pas toujours (semble-t-il) conscience.

Du côté de l'approche énonciative, les auteurs n'ont pas formulé d'hypothèses diachroniques, du moins à notre connaissance. Toutefois, c'est un fait connu que l'emploi d'emprunt, qui serait l'emploi « de base » ou «par défaut » selon cette approche, est beaucoup plus récent que les deux autres emplois canoniques d'ultérieur du passé et d'éventualité (cf. p. ex. Patard et De Mulder 2012 ou Bourova et Dendale 2014). Or l'idée que, synchroniquement, les interprétations temporelles et modales (plus anciennes) seraient des élaborations de sa valeur énonciative (plus récente) implique selon nous un allègement progressif du sens de COND vers un signifié plus abstrait. Dans ce scénario, les traits passé et ultérieur ou le trait éventuel (selon l'emploi que l'on prend comme emploi source) se seraient effacés tandis que seule la valeur énonciative se serait maintenue. En conséquence, le COND moderne aurait été réanalysé comme un marqueur plus asbtrait d'emprunt, et cela dans tous ses contextes d'emploi, y compris ceux d'ultériorité du passé et d'éventualité. L'approche énonciative impliquerait donc une hypothèse diachronique forte, celle d'une réanalyse énonciative du COND dans tous ses contextes d'emplois.

Concernant l'approche temporelle, des hypothèses diachroniques ont été avancées par Bres et al. (2012). Les auteurs partent de l'idée, défendue entre autres par Fleischman (1982: 64-66) mais que nos données diachroniques mettent en cause (section 3.2.2), que l'emploi temporel d'ultérieur du passé est premier et que les autres emplois du COND en sont directement dérivés. Du point de vue des mécanismes diachroniques, ils font appel au processus d'abstraction. Par exemple, ils présentent l'émergence de l'emploi d'emprunt (ex. Selon Sophie, Pierre participerait à la commémoration) à partir de l'emploi d'ultérieur du passé en discours indirect (ex. Sophie dit que Pierre participerait à la commémoration) comme «le passage d'une structure syntaxique de discours rapportant un acte d'énonciation antérieur (e) à un structure qui, par abstraction, le présuppose sans rien en dire (...) ${ }^{10}$ (Bres et al. 2012: 39). En conséquence, l'énonciation antérieure (e) apparaît dans l'emploi d'emprunt « sous une forme allégée de moindre intension et de plus grande extension » (ibidem).

\footnotetext{
${ }^{10} \mathrm{C}$ 'est nous qui soulignons.
} 
Sans l'admettre tout à fait, les auteurs décrivent également des restructurations du sens $d u$ COND. Ainsi, des traits centraux dans l'emploi premier temporel ne sont plus que «présupposé[s] » dans les nouveaux emplois, p. ex. le trait passé n'est plus que présupposé dans l'emploi d'emprunt où il correspond à l'antériorité de l'acte d'énonciation. Parallèlement, des traits contextuels ou des inférences sont venus «s'incorpor[er] [au] sémantisme » du COND et sont désormais des éléments «saillant[s]» (p. ex. l'inférence d'incertitude impliquée par le trait d'ultériorité dans l'emploi d'éventualité S'il se libérait, Pierre participerait à la commémoration). En bref, ce ne sont pas les mêmes traits sémantiques qui sont présupposés ou focalisés dans les différents emplois du COND. Il en faudrait peu pour que l'analyse devienne polysémiste... Les analyses que nous proposons n'en sont d'ailleurs pas très éloignées (cf. section 3.2.3.). Toutefois les auteurs sont visiblement rétifs à l'idée d'une polysémie et s'appliquent à montrer la « rémanence » du sens temporel dans les emplois dérivés du COND.

Dans la prochaine section, nous esquissons les contours d'une conception polysémique du COND (section 3.1) : selon notre hypothèse, les emplois canoniques du COND sont des constructions conventionnalisées au sens des grammaires de construction. Celle-ci s'accorde bien avec les données diachroniques, notamment avec la double origine étymologique du COND (section 3.2), et constitue, nous le verrons, une hypothèse diachronique a minima (section 3.3).

\section{Pour une approche constructionnelle et polysemiste}

\subsection{Principes}

\subsubsection{La notion de construction}

Nous rappelons les principes suivants de la théorie des grammaires de constructions (voir p. ex. Goldberg 1995 et 2006) qui sont centraux pour notre proposition :

- les unités fondamentales de la langue sont des constructions (désormais Cxn) définies comme l'association conventionnalisée d'une forme et d'un sens ;

- les configurations linguistiques complexes et abstraites (comme la construction ditransitive analysée dans Goldberg 1995) sont, en tant que Cxn, considérées comme des « signes » au même titre que les morphèmes ou les lexèmes ;

- de nombreuses Cxn sont non-compositionnelles : leur sens n'est pas fonction du sens de leurs constituants ;

- une Cxn appartient généralement à un réseau de constructions dont elle hérite en partie des propriétés syntaxiques, sémantiques, pragmatiques, tout en possédant ses traits propres.

\subsubsection{Le COND comme Cxn}

Dans ce cadre, nous faisons l'hypothèse que le COND ne correspond pas en français à une unité mais plusieurs unités linguistiques ou Cxn formant un réseau dans la compétence des locuteurs. Selon cette hypothèse, la forme $/ \mathrm{R} \varepsilon / \mathrm{du} C O N D$ est associée à un niveau schématique à trois Cxn que nous appelons désormais COND-UltPa, COND-Empr et COND-Even. Chacune de ces Cxn possède son signifié propre, qui correspond aux trois principaux « effets de sens » décrits dans la littérature : (i) ultériorité par rapport à un repère (ou un intervalle) passé pour CONDUltPa (ii) emprunt à une source énonciative secondaire (antérieure) COND-Empr et (iii) éventualité du contenu de l'énoncé (la proposition p est donnée comme ni vraie ni fausse pour le locuteur ${ }^{11}$ ) COND-Even. De plus, nous considérons que les autres effets de sens que l'on peut assigner au COND (p. ex. l'effet polémique, historique, de conjecture, d'inconcevable etc.) résultent d'une actualisation particulière de l'une des trois Cxn schématiques précitées. Selon nous, les interprétations du COND sont alors de véritables «effet de sens » contingents résultant de l'interaction du schéma constructionnel avec les apports sémantico-pragmatiques du co(n)texte.

${ }^{11}$ On peut toutefois considérer que le locuteur penche plus vers non $p$ que vers $p$ (voir Martin $1991: 90)$. 
L'interprétation finale peut alors s'analyser de façon componentielle comme une élaboration co(n)textuelle du sens de la Cxn.

Pour ce qui est du signifiant, nous faisons l'hypothèse que les trois Cxn ont en commun le signifiant /Re/, comme l'atteste l'ambiguïté du COND en contexte sous-spécifié :

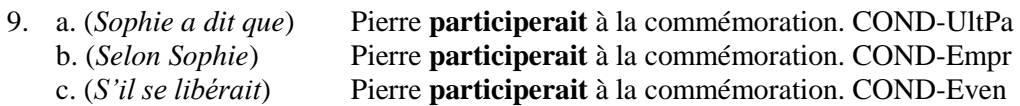

Diachroniquement, cette ambiguïté peut s'expliquer par l'érosion progressive de COND-UltPa et COND-Even (voir section 3.2.3). Notons également qu'un énoncé minimal comme Pierre participerait à la commémoration ne peut jamais recevoir d'interprétation atténuative, signe qu'il n'existe pas de construction atténuative du même niveau schématique que COND-UltPa, CONDEmpr et COND-Even.

Enfin, nous mettons en cause une analyse componentielle de $/ \mathrm{R} \varepsilon /$ comme $/ \mathrm{R} /+/ \varepsilon /$ reflétant le sémantisme du COND. En accord avec les grammaires de construction, nous suggérons que sa morphologie n'est pas forcément analysable en composants de niveau inférieur possédant chacun un signifié distinct.

Nous voudrions maintenant adopter un regard diachronique pour appuyer la thèse polysémiste.

\subsection{Regard diachronique}

\subsubsection{Corpus et méthode}

Pour la période latine, nous avons examiné une sélection de textes issus de la LIBRARY OF LATIN TEXTS (série A) éditée par Brepols. Nous nous sommes focalisée sur la période s'étendant de la fin du $2^{\text {ème }}$ siècle, où sont attestées les premiers étymons latins du COND (INF + habere conjugué à l'imparfait) ${ }^{12}$, jusqu'au $9^{\text {ème }}$ siècle, fin de la période romane. Nous avons choisi de ne prendre en compte que les occurrences avec l'ordre INF + habere ou IH pour deux raisons : (i) la morphologie du conditionnel reflète cet ordre (et non l'ordre inverse), et (ii) l'ordre $I H$ semble manifester un stade de grammaticalisation plus avancé (cf. Nocentini 2001 et Bourova 2005). Nous avons pu relever 52 occurrences sur l'ensemble des textes sélectionnés, ce qui indique que l'étymon du COND était encore très peu fréquent en latin tardif.

L'évolution du COND en français est ensuite retracée à l'aide de deux corpus construits à partir de FRANTEXT : un corpus d'ancien français comprenant les textes du $12^{\text {ème }}$ siècle et un corpus de français moderne comprenant ceux du $20^{\text {ème }}$ siècle. Nous en avons extrait les occurrences de mots réalisant les graphies du COND, accompagnées de leur contexte d'apparition. Puis nous avons procédé à un échantillonnage aléatoire ${ }^{13}$ de ces dernières pour chacun des deux corpus. Nous avons ainsi obtenu un échantillon représentatif de chaque corpus comptant chacun 200 occurrences contextualisées de COND présent.

\subsubsection{Données latines : une double origine étymologique}

Le COND trouve son origine en latin tardif dans la périphrase latine INF + habere. Selon Bourova et Tasmowski (2007:28-29, suivant Kronning 2001), celle-ci exprime principalement la nécessité aléthique "lato sensu»: il s'agit d'une nécessité d' «être », valable pour l'ensemble des mondes possibles d'un univers modal, dont aucune contingence ne permet de se soustraire. La périphrase INF + habere signifie ainsi, grâce à sa valeur aléthique, que la situation doit nécessairement se produire, qu'elle est inévitable. Le COND s'est plus précisément formé à partir d'INF + habere conjuguée à l'imparfait : INF + habebat. Toutefois, la forme INF + habebat à cette époque ne doit

${ }^{12}$ C'est à la fin du $2^{\text {ème }}$ siècle que la périphrase habere + INF est proche de la synthèse et commence à fonctionner comme un auxiliaire.

${ }^{13}$ Réalisé à l'aide de deux programmes informatiques. Nous remercions Nicolas Chollet pour l'aide apportée lors de cette étape. 
pas être considérée comme une construction autonome ayant un signifié propre (tout comme venait $d e+I N F$ par rapport à venir de $+I N F$ en français moderne), notamment parce que ses emplois sont communs aux autres formes conjuguées de INF + habere $^{14}$ et que son interprétation est tout à fait compositionnelle. En d'autres termes, «l'étymon» du COND n'est pas une unité linguistique autonome mais seulement une (ou plutôt des) instanciation(s) particulière(s) de INF + habere.

L'analyse du corpus montre que toutes les occurrences d'INF + habebat se répartissent en deux contextes d'emplois clairement distincts que l'on peut considérer comme les deux sources étymologiques du COND français : un emploi de «prédestination» (cf. Benveniste 1974), et un emploi dans les phrases hypothétiques en si.

L'emploi de prédestination est de loin le plus fréquent dans les textes ( $87 \%$ des cas) et est attesté à partir de la fin du II ${ }^{\text {ème }}$ siècle. La nécessité de l'événement est alors envisagée à partir d'un point de vue passé, presque toujours une prédiction. Cet emploi se rencontre ainsi le plus souvent dans les textes de prédicateurs chrétiens pour évoquer des événements prédits dans le passé et dont on sait qu'ils se sont réalisés :

10. [...] dominus iesus christus in eo quod puer erat $[\ldots]$ crescere et confortari habebat in eo uero quod etiam uerbum dei et deus aeternus [...] (Bède le vénérable)

'le seigneur jésus christ dans ce qui était enfant grandirait et se renforcerait (litt. devait grandir et se renforcer) dans cette vérité, qui était alors le verbe de dieu et le dieu éternel'

Le sens de nécessité aléthique se traduit par la prépondérance de certains paramètres contextuels impliquant que la situation dénotée échappe à la volonté du sujet (ce qui est logique si la situation est nécessairement vraie) : on trouve ainsi fréquemment des verbes non agentifs (p. ex. comme nasci « naître »), des sujets inanimés et la diathèse passive (11).

11. [...] saginatus carnem domini figurabat, quae ab incredulis filiis carnis abrahae propter salutem credentium immolari habebat [...]. (Grégoire d'Elvire)

"le tendre agneau figurait la chair du seigneur, laquelle serait immolée (litt. devait être immolé) par les fils incroyants de la chair d'Abraham pour le salut des croyants'

Tous ces éléments concourent, avec INF + habebat, à l'interprétation aléthique selon laquelle la situation doit inévitablement être vraie.

A côté de l'emploi de prédestination, le second emploi représente $13 \%$ des occurrences : il s'agit de l'emploi hypothétique dans la construction [si p, q]. Cet usage est attesté dans les textes à partir du $\mathrm{V}^{\text {ème }}$ siècle, époque où INF + habebat commence à concurrencer l'imparfait dans l'apodose de phrases hypothétiques éventuelles (Haverling $2010: 150$ ).

12. Sanare te habebat deus per indulgentiam, si fatereris [...].(Césaire d'Arles) 'Dieu te guérirait (à coup sûr) dans son indulgence, si tu passais aux aveux'

Selon Thomas (2012: 18-19), INF + habebat est aussi porteur dans ce contexte d'un sens de nécessité : il souligne la réalisation inévitable des événements décrits, si tant est que les conditions de la protase sont remplies. La périphrase semble donc trouver en phrase hypothétique un usage emphatique qui vient renforcer le lien conditionnel propre à la structure corrélative. Notons qu'à cette époque, INF +habebat (tout comme l'imparfait) ne réfère déjà plus au passé dans les [si $\mathrm{p}, \mathrm{q}]$. En effet, alors qu'en latin classique l'imparfait renvoie dans les hypothétiques à des situations contrefactuelles passées, il s'associe, à partir du latin tardif, à des situations non-passées, dont la

${ }^{14}$ Les mêmes contextes d'emplois sont p. ex. observés pour la périphrase au présent (INF + habet) qui donnera le futur synthétique français. 
réalisation est jugée possible mais peu probable (cf. Patard et De Mulder, 2014). Cela signifie que, même dans son premier usage dans les [si p, q], l'ancêtre du COND n'a jamais renvoyé à l'époque passée.

En conclusion, l'étymon latin du COND connaît deux emplois qui se distinguent par leur interprétation : (i) l'emploi de prédestination où INF + habebat exprime la nécessité aléthique dans le passé ; et (ii) l'emploi hypothétique où INF + habebat souligne le lien nécessaire entre une condition éventuelle et sa conséquence. Selon toute vraisemblance, ces deux usages sont la source des emplois temporel et éventuel du COND français, i.e., selon notre hypothèse, de COND-UltPa et COND-Even. Les données mettent aussi en doute le scénario de la primauté de l'emploi temporel sur les autres emplois du COND (défendue entre autres par Fleischman 1982) : le COND n'a pas une mais deux origines étymologiques distinctes. Il s'ensuit que COND-Even n'est pas dérivé de Cond-UltPa.

\subsubsection{Du latin au français moderne : scénarios diachroniques}

Le passage du latin au français est marqué par la grammaticalisation du COND à partir des deux emplois source décrits précédemment. La grammaticalisation en question illustre bien la définition qu'en donne Bybee (2010: 106) : "la création d'un nouveau morphème grammatical et d'une nouvelle construction à partir d'une instance particulière d'une construction plus ancienne ». En l'occurrence, deux emplois particuliers de INF + habere se sont autonomisés pour devenir deux unités linguistiques à part entière : les deux constructions grammaticales COND-Even et CONDUltPa. La troisième Cxn (COND-Empr) a émergé plus tard dans l'histoire du français.

\subsubsection{COND-Even}

En ancien français, COND-Even se différencie de son étymon à la fois sur le plan sémantique et syntaxique. Sémantiquement, la nécessité aléthique n'apparaît plus comme un trait saillant, le lien conditionnel (s'il existe) n'est plus vraiment interprété comme un lien de conséquence nécessaire. La valeur centrale semble désormais être celle d'éventualité : la proposition $\mathrm{p}$ est donnée comme hypothétique, i.e. ni vraie ni fausse. Certaines occurrences semblent ainsi exprimer une pure éventualité, notamment en contexte négatif où ladite éventualité est niée :

13. se set covrir / Et bien tapir por descovrir / Son compaingnon (...) / Tant s'est entremis de l'aprandre / Que nus ne l'en porroit reprendre.

'il sait se protéger ou s'aplatir, pour prendre son adversaire par surprise (...) : sur ce point, il en sait tant que nul ne pourrait lui en remontrer.' (Roman de Renart)

L'interprétation éventuelle est aussi la seule lecture possible du COND en protase puisqu'il y décrit alors la situation supposée et non la conséquence qu'elle conditionne. C'est le cas dans les relatives hypothétiques en [qui COND, COND] :

14. Ami Guischard, qui t'en fereit porter / E des granz plaies fereit tun cors saner, / Tun escientre ent[e]rei[e]s ja en ciel ?

'Ami Guichard, si l'on t'emportait et soignait tes profondes plaies, entrerais-tu tout de suite au ciel ?' (Chanson de Guillaume)

Sur le plan syntaxique, le COND s'est affranchi des phrases en [si p, q] pour s'étendre à de nouveaux contextes d'éventualité, dans d'autres structures corrélatives (14) mais aussi en dehors de celle-ci (13). On peut analyser cette évolution comme le résultat d'un double processus accompagnant la grammaticalisation de COND-Even :

- l'allègement (ou abstraction) de la Cxn latine [si p, INF + habebat $]$ qui s'émancipe sémantiquement et formellement de la structure conditionnelle,

- la réanalyse subséquente de COND-Even comme simple marqueur d'éventualité.

Notons que la grammaticalisation de COND-Even a aussi entraîné une perte de compositionnalité : comme le morphème /R/ (issu de INF + habere) était porteur de la valeur de nécessité aléthique, 
l'effacement de cette dernière a pour effet qu'il n'est plus possible d'analyser /R/ comme un composant du COND ayant son propre signifié. L'ensemble du morphème $/ \mathrm{R} \varepsilon /$ a été réanalysé comme une marque d'éventualité.

La réanalyse en marqueur d'éventualité semble ensuite confirmée par l'évolution ultérieure de COND-Even. Soit le tableau suivant qui représente l'évolution de l'expression de la condition de l'ancien français au français moderne.

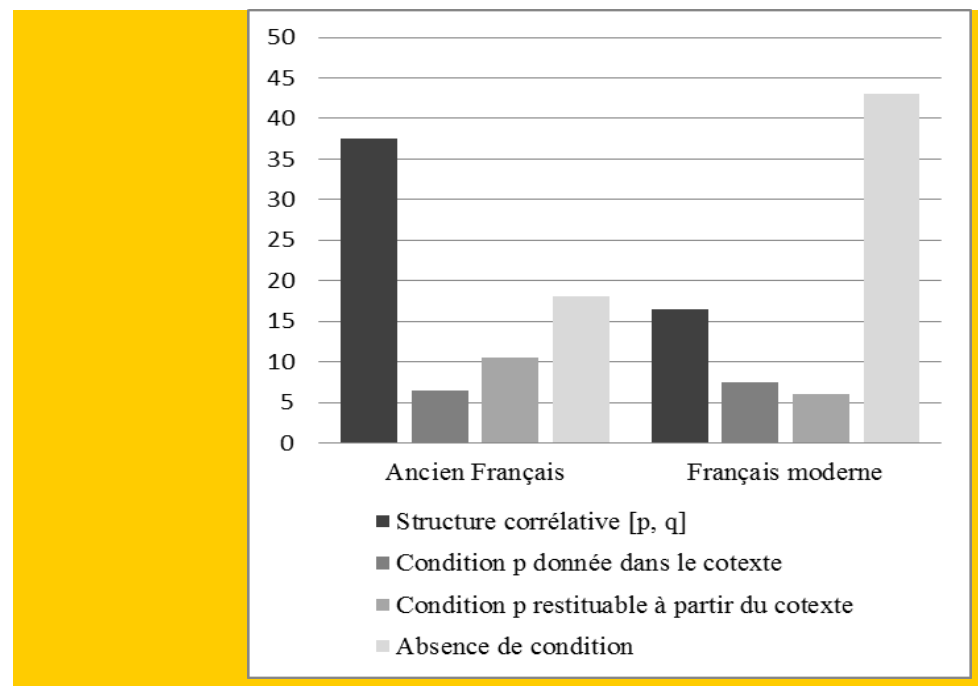

Figure 1. COND-Even et l'expression de la condition (en pourcentage)

La figure montre que l'expression de la condition par des structures corrélatives était majoritaire en ancien français (37\%), mais qu'elle a nettement reculé aujourd'hui (17\%). Dans le même temps, la présence de la proposition conditionnante $\mathrm{p}$ dans le cotexte (qu'elle soit donnée ou non telle quelle) a légèrement régressé (14\% contre $17 \%$ en ancien français). Corollairement, l'absence de proposition conditionnante exprimée ou sous-entendue, et donc, peut-on penser, l'absence de sens conditionnel, a très significativement augmenté et représente maintenant l'écrasante majorité des cas $(43 \%$ contre $18 \%$ en ancien français). Dans ce dernier cas, COND-Even s'emploie dans une grande variété de contextes : avec des verbes modaux (pouvoir, vouloir, falloir, devoir), en comparative hypothétique (ex. comme un squelette qui sortirait d'une tombe), dans des constructions spécifiques (p. ex. [ne savoir.COND], [on dire.COND], [aimer.COND mieux], [on se croire.COND]) ou schématiques ([V de + INF], ex. La police anglaise rougirait de faire partie $d u$ prolétariat.), etc.

Si l'on adopte le scénario diachronique d'Heine (2002), on peut dire que l'extension du COND à une variété de nouveaux contextes dépourvus d'interprétation conditionnelle correspond au quatrième et dernier stade du changement sémantique où la conventionnalisation du sens purement éventuel est achevée.

\subsubsection{COND-UltPa}

Dans nos données, COND-UltPas s'emploie en ancien français dans des contextes de Discours Indirect ou Indirect Libre (DI(L)) :

15. Sire, por Deu, com esfreee / Tot maintenant, sanz consoil prendre, / Dis je m'an feroie desfandre / D'un chevalier ancontre trois. (Yvain ou le Chevalier au Lion, Chrétien de Troyes) 
'Seigneur, par Dieu, dans mon effroi, j'ai aussitôt dit, sans réfléchir, que mon chevalier me défendrait contre trois adversaires.'

La formation COND-UltPa à partir de l'emploi de prédestination de l'étymon latin peut s'analyser comme le résultat de la conventionnalisation d'une inférence contextuelle d'ultériorité, qui était fréquemment attachée au sens aléthique de INF + habere. En effet, en présentant un événement (souvent télique) comme devant nécessairement avoir lieu, le locuteur implique que celui-ci doit se réaliser ultérieurement (cf. les exemples latins (10) et (11) cités supra). La répétition fréquente de ce type de contexte transitionnel, où coexistent nécessité aléthique et ultériorité (cf. les bridging contexts de Heine 2002), a pu entraîner la conventionnalisation de l'implicature d'ultériorité au détriment du sens originel aléthique. Une fois l'ultériorité conventionnalisée, la Cxn peut s'utiliser dans des contextes de DI(L) qui appellent justement cette valeur (cf. (15)). COND-UltPa est désormais compatible avec des actions contrôlables par un agent (p. ex. donner en (16)), ce qui témoigne de la disparition du sens de nécessité aléthique :

16. (...) li bons rois li avoit ja pramis, / Qant il avroit de Rome le roiaume conquis, / Q'il li donroit Melans au jor qu'il l'avroit pris (...). (Roman d'Alexande, A. Dde Paris)

'le bon roi lui avait promis il y a longtemps que, quand il aurait conquis le royaume de Rome, il lui donnerait Milan le jour où il aurait pris la cité'

En ancien français, COND-UltPa est donc devenu un véritable temps de l'indicatif marquant l'ultériorité dans le passé. Preuve en est le récent développement de son emploi « historique » dans les narrations passées, où il sert à exprimer l' « ultériorité du passé objective » (cf. Bres 2012a).

\subsubsection{COND-Empr}

COND-Empr, peu représenté dans notre corpus (6 occurrences), n'est documenté qu'à partir du $16^{\text {ème }}$ siècle (Bourova et Dendale 2013). Nous faisons l'hypothèse qu'il trouve son origine dans l'usage de COND-UltPa en DI(L). La filiation diachronique entre les deux Cxn repose sur plusieurs arguments. D'abord, elles sont toutes deux contextuellement associées à une énonciation antérieure : dans un cas, un discours passé dénoté par un verbe énonciatif enchâssant (CONDUltPa) et, dans l'autre cas, la donation initiale présupposée d'une information reprise (CONDEmpr). De plus, l'interprétation ultérieure constitutive de COND-UltPa est aussi compatible avec COND-Empr (Ex. Selon Sophie, Pierre participerait demain à la commémoration). Enfin, les premières occurrences avec valeur d'emprunt apparaissent très souvent en discours indirect :

17. On nous escrit que les Turcs ont esté n'guéres défaits par les Moscovites en deux batailles : où les premiers auraient perdu soixante $\&$ dix mille hommes. (Gazette 109, 7 septembre 1641 < Bourova et Dendale $2013: 191$ )

Le mécanisme à l'œuvre dans la formation de COND-Empr peut être décrit, suivant le scénario diachronique de Heine (2002), comme l'intégration du sens contextuel d'emprunt présupposé par le DI (une information rapportée est nécessairement empruntée) dans le sémantisme de la Cxn. Parallèlement, la valeur d'ultériorité s'efface (mais peut-être réintroduite co(n)textuellement, cf. supra) et l'énonciation antérieure, reléguée à l'arrière-plan, n'est plus que présupposée. En l'absence de référence passée assertée, l'ancrage temporel se fait dans le présent ou le futur (Ex. Selon Sophie, Pierre participerait à la commémoration, voir aussi Bres 2012b). La Cxn a ainsi été réanalysée comme un authentique marqueur évidentiel exprimant l'information empruntée.

\subsubsection{COND et atténuation}

Dès l'ancien français, le COND peut revêtir une nuance atténuative, souvent dans des corrélations hypothétiques en si qui permettent de présenter indirectement un acte de langage potentiellement 
menaçant pour l'interlocuteur. Par exemple, le locuteur peut conditionner la réalisation de l'acte visé au bon plaisir de l'interlocuteur :

18. s'il vous plesoit, par autre voie a sauveté vous en menroie. (Le Roman de Thèbes) 'si vous y consentiez, je vous conduirais dans un endroit sûr par un autre chemin.'

ou bien exprimer un désir subordonné à des prémisses favorables (qui dépendent aussi de l'interlocuteur) :

19. « La, voldroie, fet il, aler, a l'ermite, se ge savoie tenir le santier et la voie. (...)» (Perceval, C. de Troyes)

' Je voudrais aller là-bas, fit-il, chez l'ermite, si je connaissais le sentier et le chemin à suivre »'

Dans tous les cas observés, l'atténuation n'apparaît que comme une implication découlant de la valeur conditionnelle et/ou éventuelle portée par l'énoncé au COND. Il n'y a donc pas lieu ici de parler de Cxn à part entière, il s'agit d'un « effet de sens » particulier de COND-Even.

Les données du français moderne montrent que l'usage de COND-Even à nuance atténuative s'est aussi libéré des [si p, q], suivant ainsi la tendance générale de la Cxn (cf. section 3.2.3.1). Le mouvement d'expansion hors corrélation hypothétique semble de plus avoir permis la formation de micro-constructions d'atténuation où le sens d'éventualité est mis à l'arrière-plan, p. ex [vouloirp1/2.COND], [aimer- $1 / 2 . C O N D])$ :

20. Yvonne, je voudrais savoir si tu te moques de moi. (Les Parents terribles, J. Cocteau)

Le fait qu'il s'agisse de constructions lexicalement spécifiques est incompatible avec l'idée d'un COND à sens atténuatif, ce qui supposerait au contraire une absence de restriction lexicale sur le verbe. On peut donc admettre qu'il n'existe pas de COND-Att.

\subsubsection{Bilan provisoire}

Quelles conclusions (provisoires) pouvons-nous tirer des données diachroniques concernant la monosémie ou la polysémie du COND en français moderne ? Il apparaît que les approches monosémistes, qu'elles soient modales ou temporelle, impliquent des hypothèses diachroniques fortes, c'est-à-dire des restructurations sémantiques majeures.

L'approche énonciative-inactuelle suppose un processus d'abstraction sémantique à partir des différentes interprétations du COND conduisant à sa réanalyse énonciative dans tous ses emplois : la valeur d'emprunt, absente dans «l'étymon » latin et secondaire en ancien français (seulement présente en arrière-plan), aurait ainsi supplanté les autres signifiés plus spécifiques au cours de l'évolution du français. Etant donné que cette valeur n'est vraiment saillante que dans l'emploi évidentiel, très peu fréquent, ce scénario paraît peu probable.

L'approche temporelle est quant à elle principalement confrontée à la filiation entre l'emploi temporel et l'emploi d'éventualité. Comme les données diachroniques ne confirment pas la primauté de l'emploi temporel postulé par Fleischman (1982), mais indique une double origine étymologique, il reste à ce type d'approche de supposer une réanalyse temporelle après coup de l'emploi d'éventualité, c'est-à-dire après la grammaticalisation du COND et l'incorporation du trait d'ultériorité dans son sémantisme. Ce scénario ne peut être en soi écarté, mais il constitue un changement sémantique majeur (et inédit ?) où l'éventualité serait réinterprétée en ultériorité du passé.

A contrario, le scénario correspondant à notre approche polysémiste-constructionnelle (cf. 3.2.3) constitue plutôt une hypothèse a minima. Deux instances particulières d'une construction latine (INF + habere) se sont autonomisées et grammaticalisées pour devenir deux constructions à part entière en ancien français : COND-Even et COND-UltPa ; puis COND-Empr a émergé à partir de COND-UltPa. Ce scénario n'implique pas de réinterprétation sémantique majeure 
(contrairement aux autres scénarios précédents), mais des glissements de focus avec des sens secondaire(s) (inférés ou co(n)textuels) qui sont devenus progressivement saillants et centraux. Toutefois, nous en convenons, cela ne signifie pas que les réinterprétations majeures évoquées précédemment n'ont pas eu lieu. C'est là la limite de l'argument diachronique, l'explication est toujours dans une certaine mesure spéculative.

\section{En guise de conclusion}

L'analyse polysémiste et constructionnelle que nous avons esquissée doit être encore affinée en synchronie et confirmée par des études diachroniques. Toutefois, comme nous l'avons souligné, il n'existe aucun argument décisif, théorique ou diachronique, en faveur de la polysémie du COND. Seule des expérimentations psycholonguistiques permettraient (éventuellement) d'avancer sur cette question.

Néanmoins notre analyse polysémiste permet déjà de lever certaines difficultés rencontrées par les approches monosémistes. Par exemple, le vieux débat « COND : temps ou mode ? » trouve une issue très simple avec un COND polysémique: COND-UltPa est un véritable temps verbal, COND-Even un marqueur modal (mais s'agit-il d'un mode ?) et COND-Empr un authentique marqueur évidentiel. Ce type d'approche soulève aussi des nouvelles interrogations: sur la polysémie des autres temps verbaux (comme l'imparfait), sur notre conception et les critères à adopter pour définir «les temps de l'indicatif», sur la question des modes etc., et plus généralement sur la sémantique des formes verbales. Autant de questions qui méritent, selon nous, d'être posées sans a priori théorique (monosémiste ou polysémiste). 


\section{Références}

Abouda L. (2001), «Les emplois journalistique, polémique et atténuatif du conditionnel », in P. Dendale, L. Tasmowski (éds.), Le conditionnel en français, Paris : Klincksieck, 277294.

Abouda L. (2016), «Conséquences morphologiques du traitement du conditionnel comme un temps de l'indicatif », 5e Congrès Mondial de Linguistique Française, Juil. 2016, Tours, Actes du CMLF 2016.

Azzopardi S. (2011), Le futur et le conditionnel: valeur en langue et effets de sens en discours. Analyse contrastive espagnol/français, Thèse de doctorat, Montpellier: Université Paul-Valéry Montpellier III.

Barbazan M. (2006), Le temps verbal. Dimensions linguistiques et psycholinguistiques, Toulouse : Presses universitaires du Mirail.

Benveniste É. (1974), Problèmes de linguistique générale, II, Paris : Gallimard.

Berthonneau A.-M., Kleiber G. (1994), «Imparfaits de politesse : rupture ou cohésion ? », Travaux de linguistique 29, 59-92.

Bourova V. (2005), «A la recherche du 'conditionnel latin': les constructions 'Infinitif + forme habere' examinées à partir d'un corpus électronique », in C. D. Pusch, J. Kabatek, W. Raible (éds), Romanistische Korpuslinguistik II, Tübingen : Gunter Naar Verlag, 303316.

Bourova V., Dendale P. (2013), «Serait-ce un conditionnel de conjecture? Datation, évolution et mise en relation de deux conditionnels à valeur évidentielle », Cahiers Chronos 26, 183-200.

Bourova V., Tasmowski L. (2007), «La préhistoire des futurs romans. Ordre des constituants et sémantique », Cahiers Chronos 19, 25-41.

Bres J. (2003), « Non, le passé simple ne contient pas l'instruction [+ progression] », Cahiers Chronos 11, 99-112.

Bres J. (2009), « Dialogisme et temps verbaux de l'indicatif », Langue française 163, 21-39.

Bres J. (2012a), «Conditionnel et ultériorité dans le PASSÉ : de la subjectivité à l'objectivité », SHS Web of Conferences 1 ( $^{\text {ème }}$ Congrès Mondial de Linguistique Française), 1719-1730.

Bres J. (2012b), «Robert aurait pris sa retraite et passerait du bon temps... Du conditionnel dit journalistique », in Maury-Rouan, Cl. (éd.), Regards sur le discours, Aix-en-Provence : Presses de l'université de Provence, 15-33.

Bres J., Azzopardi S., Sarrazin S. (2012), «Le conditionnel en français : énonciation, ultériorité dans le passé et valeurs modales ", Faits de langues 40, 37-43.

Bybee J. L. (2010), Language, usage and cognition, Cambridge: Cambridge University Press.

Camussi-Ni M. A. (2006), Analyse formelle et conceptuelle des formes verbales du français contemporain: A la croisée du passé simple et de l'imparfait, du futur et du conditionnel, les concepts"+/-potentiel" et"+/-défini", Thèse de doctorat, Rennes : Université Rennes 2.

Caudal P., Vetters C. (2005), "Un traitement conjoint du conditionnel, du futur et de l'imparfait : les temps comme des fonctions d'actes de langage », Cahiers Chronos 12, 109-124.

Damourette J., Pichon É. (1911-1936/1970), Des mots à la pensée, vol. 5, Paris : D’Artrey.

Dendale P. (2001), «Les problèmes linguistiques du conditionnel en français », in Dendale P., Tasmowski L. (éds), Le conditionnel en français, Paris : Klincksieck, p. 7-18.

Fleischman S. (1982), The future in thought and language, Cambridge: Cambridge University Press. 
Goldberg A. (1995), Constructions: A construction grammar approach to argument structure, Chicago: University of Chicago Press.

Goldberg A. (2006), Constructions at work. The nature of generalization in language, Oxford: Oxford University Press.

Gosselin L. (1996a), «Le traitement de la polysémie contextuelle dans le calcul sémantique », Intellectica 22, 93-117.

Gosselin L. (1996b), Sémantique de la temporalité en français: un modèle calculatoire et cognitif du temps et de l'aspect, Louvain-la-Neuve : Duculot.

Gosselin L. (1999), «Les valeurs de l'imparfait et du conditionnel dans les systèmes hypothétiques », Cahiers chronos 4, 29-51.

Gosselin L. (2001), «Relations temporelles et modales dans le «conditionnel journalistique », in Dendale P., Tasmowski L. (éds), Le conditionnel en français, Metz, Paris : Université de Metz, 45-66.

Guillaume G. (1929/1970), Temps et verbe, Paris : Champion.

Haillet P. P. (2002), Le conditionnel en français : une approche polyphonique, Gap : Ophrys.

Haillet P. (2003), «Représentations discursives, point(s) de vue et signifié unique du conditionnel », Langue française 138, 35-47.

Heine B. (2002), « On the role of contexts in grammaticalization », in Wischer, I., Diewald, G. (éds), New Reflections on Grammaticalization, Amsterdam: John Benjamins, 83-101.

Korzen H., Nølke H. (2001), «Le conditionnel : niveaux de modalisation », in Dendale P., Tasmowski L. (éds), Le conditionnel en français, Metz, Paris : Université de Metz, 125145.

Kronning H. (2001), «Pour une tripartition des emplois du modal devoir », Cahiers chronos 8, 67-84.

Kronning H. (2002), «Le conditionnel 'journalistique' : médiation et modalisation épistémiques », Romansk forum 16 (2), 561-575.

Martin R. (1991), « Types de procès et systèmes hypothétiques. De l'aspect de re à l'aspect de dicto $»$, Travaux de linguistique et de philologie XXIX, 87-95.

Moeschler J., Reboul A. (2001), «Conditionnel et assertion conditionnelle», in Dendale P., Tasmowski L. (éds), Le conditionnel en français, Paris : Klincksieck, 145-167.

Nocentini A. (2001), «La genesi del futuro et del condizionale sintetico romanzo», Zeitschrift für Romanische Philologie 117 (3), 367-401.

Patard A., (2007), L'un et le multiple. Valeur en langue et usages de l'imparfait en discours, Thèse de doctorat, Montpellier : Université Paul-Valéry - Montpellier III.

Patard A., De Mulder W. (2012), «L'évolution des usages du conditionnel en français », Faits de langues 40, 29-36.

Patard A., De Mulder W. (2014), « Aux origines des emplois modaux de l'imparfait. Le cas de l'emploi hypothétique et de l'emploi contrefactuel », Langages 193, 33-47.

Thomas J.-F. (2012), «Ultériorité dans le passé et éventualité en latin », Faits de langues 40, 13-20.

Vet C., Kampers-Manhe B. (2001), «Futur simple et future du passé : leurs emplois temporels et modaux », in Dendale P., Tasmowski L. (éds), Le conditionnel en français, Metz, Paris : Université de Metz, 89-104.

Vetters C. (2001), «Le conditionnel : ultérieur du non-actuel », in Dendale P., Tasmowski L. (éds), Le conditionnel en français, Paris : Klincksieck, 169-207.

Victorri B., Fuchs C. (1996), La polysémie - Construction dynamique du sens, Paris: Hermès. 
Wilmet M. (2001), «L'architectonique du conditionnel », in Dendale P., Tasmowski L. (éds), Le conditionnel en français, Paris : Klincksieck, 21-44.

Wilmet M. (2003), Grammaire critique du français, Bruxelles : Duculot. 